Our Nature (2007)5:14-20

\title{
Rhino-Fodders in Jaldapara Wildlife Sanctuary in Duars of West Bengal, India
}

\author{
Chandrâ Ghosh and A. P. Das \\ Taxonomy and Environmental Biology Laboratory, Department of Botany, North Bengal University, Darjeeling \\ 734430, West Bengal, India \\ E-mail: amichandra07@gmail.com; dasap.nbu@gmail.com
}

Received: 1.12.2007, Accepted: 12.12.2007

\begin{abstract}
Jaldapara Wildlife Sanctuary is the second largest in situ conservatory in India for Rhinoceros unicornis L. As much as 57 species of Rhino-fodders have been recorded from the sanctuary and the list is dominated with the record of 34 species (i.e. $59.65 \%$ ) from Poaceae itself. It is followed by five species of Cyperaceae and three species of Euphorbiaceae. Another 13 families are represented with two or one species only. These plants have been categorised into three groups (1) Most Preferred, 15 spp., (2) Preferred, 38 spp. and (3) Stress-time fodders, 4 spp. Local names, habit and edible parts of these plants have been provided in this article.
\end{abstract}

Keywords: Rhino fodder, Jaldapara Wildlife Sanctuary.

\section{Introduction}

Jaldapara Wildlife Sanctuary (JWLS) is the second largest natural home after Kajiranga National Park in Assam for great Indian one-horned Rhinoceros (Rhinoceros unicornis L.) and is one of the prestigious sanctuaries of West Bengal. This unique trouser shaped sanctuary (Figure 1) is situated in the Duars at the foot of the Darjeeling part of the Eastern Himalaya and falls in the district of Jalpaiguri (West Bengal, India), located in between $25^{\circ} 58^{\prime} \mathrm{N}$ and $27^{\circ} 45^{\prime} \mathrm{N}$ latitudes and $89^{\circ} 08^{\prime} \mathrm{E}$ and $89^{\circ} 55^{\prime}$ E longitudes, falls under the jurisdiction of Coochbehar Wildlife Forest Division.

This Sanctuary is situated at the foothill region which is popularly referred as Duars. In 1941 it was first declared as a 'Game Sanctuary' with an area of $99.5 \mathrm{sq} \mathrm{km}$. But, later on it was extended twice in 1976 and in 1990 to achieve its present area of 216.5 sq km (Pandit 1996; Anonymous, 1997; Das et al., 2003). For administrative purposes, Sanctuary has been divided into 07 forest ranges, 25 forest Beats and 68 compartments.

Most Rhino concentrated areas of the Sanctuary are East, West and North Ranges. They visit occasionally in some areas of Chilapata and Kodalbasti Ranges. But Rhinos never visit any part of South and Nilpara Ranges.

Its topographical situation and environmental factors made itself a beautiful natural habitat not only for Rhinos but also for an amazing variety of wild flora and fauna. As it is situated in the Duars at the foot of the Darjeeling hills, it becomes a part of the IUCN recognised Himalayan Hotspot. Darjeeling region is situated nearly at the central part of the Eastern Himalaya and is famous for its fabulous biodiversity. 
Chandra Ghosh and A.P. Das / Our Nature (2007)5: 14-20
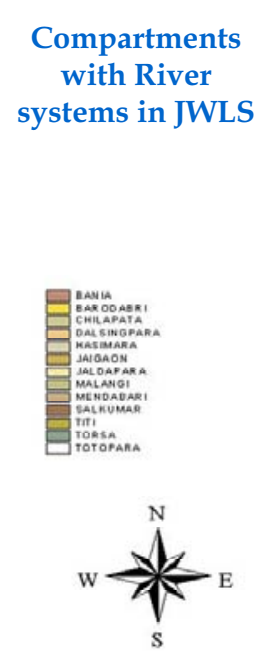

River System in

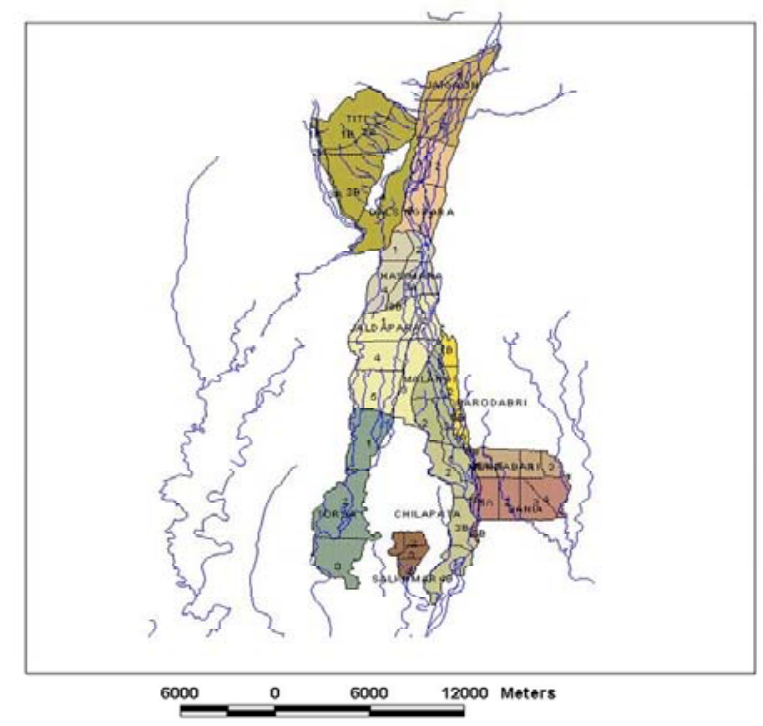

Figure 1. The map of Jaldapara Wildlife Sancuary.

Like hills, Duars and Terai are also equally rich in their biodiversity.

The basic vegetation structure of this region is mixed deciduous forest (Champion and Seth, 1968). Recent workers (Banerjee, 1993; Pandit, 1996; Anonymous, 1997; WII 1997; Das et al., 2003) recognised the formation of following types of vegetation in JWLS: Riverine Forests, Sal Forests, Wet Mixed Forests, Semi-evergreen Forests, Evergreen Forests, Riverine Grasslands, Savannah Grasslands, Open low lying Herbland, Hydrophytic vegetation, etc. A good number of threatened and endemic species as well as wide range of timber yielding, fodder, food, medicinal and other types of NTFP producing plants (Pandit, 1996; Das et al., 2003; Pandit et al., 2004) grow in the sanctuary. All strata in vegetation are floristically rich and have created the situation very much congenial for evolution.

\section{Materials and methods}

Random survey was made for rhino-fodders during 2002 and 2007 in different rhino concentrated regions of JWLS.

Collected specimens were processed and identified through conventional techniques (Jain and Rao, 1977) in the taxonomy laboratory of the Department of Botany, North Bengal University and matched at CAL and stored in the NBU-Herbarium.

\section{Results and discussion}

Rhinos are voracious consumers. Their food habit is very selective and they forage only on the young twigs of their edible plants. The list of edible plants for Rhinos is quite short and that too is dominated with grasses. 
Even they consume very little of the sedges, other monocotyledonous plants and few dicotyledonous plants.

Banerjee (1993), Pandit (1996) and Anonymous (1997) provided lists of 49 fodder plants as Rhino-fodder. Of them, later two workers have not made any modification in the list. Whereas Das et al. in 2003 reported 58 species of Rhino-fodders.

Type of Fodders: Considering the likeliness or the preference different identified Rhinofodders can be categorised into three distinct groups:

\section{Most Preferred Fodders}

Among the recorded Rhino fodders, 15 species can be regarded as most preferred ones. These are mostly grasses and are available mainly in open and grassland vegetations. These are growing in enormous quality and are supplying major bulk of the food for Rhinos and also to other herbivores of the sanctuary. Table 1 presents the list of most preferred Rhino-fodders:

It is interesting to note that both the plant Ikra and Kasia or Kash (Table 1; Sl. No. 12 and 13) have been identified as Saccharum spontaneum though there are some morphological differences. It is expected that on further study differences at varietals level could be established.

\section{Preferred Fodders}

As much as 38 species are categorised as preferred Rhino-fodder. The degree of preference varies greatly during browsing. When a Most Preferred fodder is available in good quality, these plants are generally avoided by this giant herbivore. Table 2 presented the list of preferred Rhino-fodders.

\section{Stress-time Fodders}

Only 4 species (Table 3 ) are categorised as stress-time Rhino fodders. These plants are available in varying quantity but are generally not browsed by Rhinos when most of the preferred fodders are in good supply. But during dry season, when supply of major fodders are nil or nominal, these plants are then browsed by Rhinos.

An analysis (Table 5) of the recorded Rhino-fodders reveals that this giant herbivore prefers grasses in general as out of 57 plants 34 species (i.e., 59.65\%) are from Poaceae. And, out of 15 most preferred species only one is from Zingiberaceae (Alpinia nigra) and all others are grasses. Nearly a similar situation is for 'Preferred' group of fodders where out of 38 species 20 (i.e. 52.63\%) are grasses. Poaceae is followed by Cyperaceae (five species; all 'Preferred') and Euphorbiaceae (three species; all 'Preferred') (Table 4). Rhinos eat flowers of Bombax ceiba much eagerly. Flowers of this species are available for a very short period but at a time when there is much scarcity of fodder in the sanctuary. Flowers of Oroxylum indicum are also equally liked by Rhinos but, on the contrary, the species flowers for a much longer period and at a time when there is no dearth of fodder in the sanctuary.

The overall picture is that the recorded 57 species of Rhino-fodder from JWLS are represented by 16 angiospermic families (Table 5). There is no doubt that the giant animal prefers the grasses most and out of 57 recorded fodders 34 or $59.65 \%$ species belongs to Poaceae (Figure 2).

\section{Conclusion}

Rhinoceros unicornis $\mathrm{L}$. is the keystone species of JWLS and their population is increasing quickly. So, for the conservation of this species, it is necessary to increase 
Chandra Ghosh and A.P. Das / Our Nature (2007)5: 14-20

Table 1. Most Preferred Rhino-fodders in JWLS.

\begin{tabular}{|c|c|c|c|c|c|}
\hline $\begin{array}{l}\text { Sl. } \\
\text { No. }\end{array}$ & Plant Name & Family & Local Name & Habit & Edible Parts \\
\hline 1. & $\begin{array}{l}\text { Alpinia nigra (Gaertner) B.L. } \\
\text { Burtt }\end{array}$ & Zingiberaceae & Purundi & $\begin{array}{l}\text { Medium, } \\
\text { shoots few }\end{array}$ & $\begin{array}{l}\text { New leaves \& } \\
\text { pseudostem }\end{array}$ \\
\hline 2. & $\begin{array}{l}\text { Arundinella bengalensis } \\
\text { (Sprengel) Druce }\end{array}$ & Poaceae & Chhoto Jharu & Medium grass & $\begin{array}{l}\text { Tips with new } \\
\text { leaves }\end{array}$ \\
\hline 3. & Arundo donax $\mathrm{L}$. & Poaceae & Nol & Medium grass & $\begin{array}{l}\text { Tips with new } \\
\text { leaves }\end{array}$ \\
\hline 4. & $\begin{array}{l}\text { Axonopus compressus (Sw.) P. } \\
\text { Beauvois }\end{array}$ & Poaceae & $\begin{array}{l}\text { Chhoto } \\
\text { Chepti }\end{array}$ & $\begin{array}{l}\text { Small } \\
\text { prostrate } \\
\text { grass } \\
\end{array}$ & Whole plant \\
\hline 5. & $\begin{array}{l}\text { Cymbopogon jwarancusa } \\
\text { (Jones) Schultes }\end{array}$ & Poaceae & $\begin{array}{l}\text { Chhoto } \\
\text { Gandali }\end{array}$ & $\begin{array}{l}\text { Medium } \\
\text { bushy }\end{array}$ & $\begin{array}{l}\text { Tips with new } \\
\text { leaves }\end{array}$ \\
\hline 6. & $\begin{array}{l}\text { Imperata cylindrica }(\mathrm{L} .) \\
\text { Räusch. }\end{array}$ & Poaceae & Thatch, Kush & Small grass & New leaves \\
\hline 7. & $\begin{array}{l}\text { Saccharum arundinaceum } \\
\text { Retzius }\end{array}$ & Poaceae & Madhua & Tall grass & $\begin{array}{l}\text { Tips with new } \\
\text { leaves }\end{array}$ \\
\hline 8. & $\begin{array}{l}\text { Saccharum bengalense } \\
\text { Retzius }\end{array}$ & Poaceae & --- & Tall grass & $\begin{array}{l}\text { Tips with new } \\
\text { leaves }\end{array}$ \\
\hline 9. & $\begin{array}{l}\text { Saccharum longisetosum var. } \\
\text { hookeri (Hackel) Bor }\end{array}$ & Poaceae & Ekra & Tall grass & $\begin{array}{l}\text { Tips with new } \\
\text { leaves }\end{array}$ \\
\hline 10. & $\begin{array}{l}\text { Saccharum longisetosum } \\
\text { (Anderson) V. Naray. var. } \\
\text { longisetosum }\end{array}$ & Poaceae & Ekra & Tall grass & $\begin{array}{l}\text { Tips with new } \\
\text { leaves }\end{array}$ \\
\hline 11. & $\begin{array}{l}\text { Saccharum narenga (Nees ex } \\
\text { Steudel) Hackel }\end{array}$ & Poaceae & Dhadda & Tall grass & $\begin{array}{l}\text { Tips with new } \\
\text { leaves }\end{array}$ \\
\hline 12. & Saccharum spontaneum $\mathrm{L}$. & Poaceae & Ikra & Medium grass & New leaves \\
\hline 13. & Saccharum spontaneum L. & Poaceae & Kasia, Kash & Medium grass & New leaves \\
\hline 14. & $\begin{array}{l}\text { Themeda arundinacea } \\
\text { (Roxburgh) Ridley }\end{array}$ & Poaceae & Baro chepti & Tall grass & $\begin{array}{l}\text { Tips with new } \\
\text { leaves }\end{array}$ \\
\hline 15. & $\begin{array}{l}\text { Thysanolena latifolia } \\
\text { (Roxburgh ex Horrem) Honda }\end{array}$ & Poaceae & $\begin{array}{l}\text { Jharu, } \\
\text { Ambrosa }\end{array}$ & $\begin{array}{l}\text { Medium } \\
\text { bushy }\end{array}$ & $\begin{array}{l}\text { Tips with new } \\
\text { leaves }\end{array}$ \\
\hline
\end{tabular}

Table 2. Preferred Rhino-fodders in JWLS.

\begin{tabular}{|l|l|l|l|l|l|}
\hline $\begin{array}{c}\text { SI. } \\
\text { No. }\end{array}$ & \multicolumn{1}{|c|}{ Plant Name } & \multicolumn{1}{|c|}{ Family } & \multicolumn{1}{c|}{$\begin{array}{c}\text { Local } \\
\text { Name }\end{array}$} & \multicolumn{1}{c|}{ Habit } & Edible Parts \\
\hline 01. & Acacia catechu (L.f.) Willdenow & Mimosaceae & Khair & Tree & Young shoot \\
\hline 02. & Alpinia calcarata Roscoe & Zingiberaceae & --- & $\begin{array}{l}\text { Rhizomatous } \\
\text { herb }\end{array}$ & $\begin{array}{l}\text { Leaf, pseudostem, } \\
\text { inflorescence }\end{array}$ \\
\hline 03. & $\begin{array}{l}\text { Arundinella decempedalis } \\
\text { (Kuntze) Janowski }\end{array}$ & Poaceae & --- & $\begin{array}{l}\text { Suffrutescent } \\
\text { tall herb }\end{array}$ & $\begin{array}{l}\text { Young leaf, } \\
\text { inflorescence }\end{array}$ \\
\hline 04. & Arundinella nepalensis Trnius & Poaceae & --- & $\begin{array}{l}\text { Suffrutescent } \\
\text { tall herb }\end{array}$ & $\begin{array}{l}\text { Young leaf, } \\
\text { inflorescence }\end{array}$ \\
\hline 05. & Brachiaria ramosa (L.) Stapf & Poaceae & --- & Annual herb & Shoot, inflorescence \\
\hline 06. & Bridelia retusa (L.) Sprengel & Euphorbiaceae & Datan & Tree & Young shoot \\
\hline 07. & $\begin{array}{l}\text { Chrysopogon aciculatus } \text { (Retzius) } \\
\text { Trinius }\end{array}$ & Poaceae & Chorkanta & Annual herb & Young Shoot \\
\hline 08. & Coix aquatica Roxburgh & Poaceae & & Annual herb & Shoot \\
\hline 09. & Coix lachryma-jobi L. & Poaceae & Malagadi & Annual herb & Young leaf \\
\hline
\end{tabular}


Chandra Ghosh and A.P. Das / Our Nature (2007)5: 14-20

\begin{tabular}{|c|c|c|c|c|c|}
\hline 10. & $\begin{array}{l}\text { Curculigo capitulata (Loureiro) } \\
\text { Herbert }\end{array}$ & Hypoxidaceae & Bansh pata & Perennial herb & $\begin{array}{l}\text { Young leaf, } \\
\text { inflorescence }\end{array}$ \\
\hline 11. & $\begin{array}{l}\text { Cymbopogon flexuosus var. } \\
\text { sikkimensis Bor }\end{array}$ & Poaceae & $\begin{array}{l}\text { Baro } \\
\text { Gandali }\end{array}$ & $\begin{array}{l}\text { Suffrutescent } \\
\text { tall herb }\end{array}$ & $\begin{array}{l}\text { Young leaf, } \\
\text { inflorescence }\end{array}$ \\
\hline 12. & $\begin{array}{l}\text { Cymbopogon khasianus (Muro ex } \\
\text { Hackel) Stapf ex Bor }\end{array}$ & Poaceae & $\begin{array}{l}\text { Baro } \\
\text { Gandali }\end{array}$ & $\begin{array}{l}\text { Suffrutescent } \\
\text { tall herb }\end{array}$ & $\begin{array}{l}\text { Young leaf, } \\
\text { Inflorescence }\end{array}$ \\
\hline 13. & Cynodon dactylon (L.) Persoon & Poaceae & Durba & $\begin{array}{l}\text { Perennial } \\
\text { runner }\end{array}$ & Young shoot \\
\hline 14. & Cyperus pangorei Rottboel & Cyperaceae & --- & Annual herb & Young shoot \\
\hline 15. & Cyperus pilosus Vahl & Cyperaceae & --- & Annual herb & Young shoot \\
\hline 16. & Dalbergia sissoo DC. & Papilionaceae & Sissoo & Tree & Young leaf \\
\hline 17. & Eleusine indica $(\mathrm{L}$.$) Gaertner$ & Poaceae & Marua & Annual herb & Young shoot \\
\hline 18. & Ficus hispida L.f. & Moraceae & Khoksa & Tree & Young leaf \\
\hline 19. & Fimbristylis rigidula Nees & Cyperaceae & --- & Annual herb & Young shoot \\
\hline 20. & Helicteres hirsuta Loureiro & Sterculiaceae & --- & Tree & Young leaf \\
\hline 21. & Helicteres isora $\mathrm{L}$. & Sterculiaceae & Fersa & Shrub & Young leaf \\
\hline 22. & $\begin{array}{l}\text { Litsea monopetala (Roxburgh) } \\
\text { Persoon }\end{array}$ & Lauraceae & Kutmero & Tree & Young leaf \\
\hline 23. & $\begin{array}{l}\text { Macaranga denticulata (Blume) } \\
\text { Mueller }\end{array}$ & Euphorbiaceae & Malata & Tree & Young leaf \\
\hline 24. & $\begin{array}{l}\text { Mallotus philippensis (Lamarck) } \\
\text { Mueller }\end{array}$ & Euphorbiaceae & Sindure & Tree & Young leaf \\
\hline 25. & Nasturtium officinale Brown & Cruciferae & $\begin{array}{l}\text { Alua } \\
\text { Ghash }\end{array}$ & Annual herb & Leaf, Inflorescence \\
\hline 26. & $\begin{array}{l}\text { Oplismenus burmannii (Retzius) } \\
\text { P. Beauvois }\end{array}$ & Poaceae & $\begin{array}{ll}-- \\
\end{array}$ & Annual herb & Young shoot \\
\hline 27. & $\begin{array}{l}\text { Oplismenus compositus (L.) P. } \\
\text { Beauvois }\end{array}$ & Poaceae & --- & Annual herb & Young shoot \\
\hline 28. & Oroxylum indicum (L.) Ventenat & Bignoniaceae & Totola & Tree & Flower \\
\hline 29. & $\begin{array}{l}\text { Oryza meyeriana (Zoll. \& Moritzi) } \\
\text { Baillon }\end{array}$ & Poaceae & --- & Annual herb & Young shoot \\
\hline 30. & Panicum auritum Presl ex Nees & Poaceae & --- & Annual herb & $\begin{array}{l}\text { Young leaf, } \\
\text { Inflorescence }\end{array}$ \\
\hline 31. & Paspalum scrobiculatum $\mathrm{L}$. & Poaceae & $\begin{array}{l}\text { Marua } \\
\text { Ghash }\end{array}$ & Annual herb & Young Shoot \\
\hline 32. & $\begin{array}{l}\text { Schizachyrium brevifolium (Sw.) } \\
\text { Nees ex Büse }\end{array}$ & Poaceae & --- & Annual herb & Young Shoot \\
\hline 33. & Scleria terrestris (L.) Fassett & Cyperaceae & $\begin{array}{l}\text { Kella } \\
\text { Ghash }\end{array}$ & Annual herb & $\begin{array}{l}\text { Young Leaf, } \\
\text { Inflorescence }\end{array}$ \\
\hline 34. & Scleria levis Retzius & Cyperaceae & $\begin{array}{l}\text { Kella } \\
\text { Ghash }\end{array}$ & Annual herb & $\begin{array}{l}\text { Young Leaf, } \\
\text { Inflorescence }\end{array}$ \\
\hline 35. & Setaria palmifolia (J. König) Stapf & Poaceae & Banshpata & Annual herb & $\begin{array}{l}\text { Young Leaf, } \\
\text { Inflorescence }\end{array}$ \\
\hline 36. & $\begin{array}{l}\text { Sporobolus fertilis (Steudel) } \\
\text { Clayton }\end{array}$ & Poaceae & --- & Annual herb & $\begin{array}{l}\text { Young Leaf, } \\
\text { Inflorescence }\end{array}$ \\
\hline 37. & Tripsacum laxum Nash & Poaceae & $\begin{array}{l}\text { Bhutta } \\
\text { ghash }\end{array}$ & Perennial herb & Young Leaf \\
\hline 38. & Vetiveria zizanoides (L.) Nash & Poaceae & Bena & $\begin{array}{l}\text { Suffrutescent } \\
\text { tall herb }\end{array}$ & $\begin{array}{l}\text { Young Leaf, } \\
\text { Inflorescence }\end{array}$ \\
\hline
\end{tabular}


Chandra Ghosh and A.P. Das / Our Nature (2007)5: 14-20

Table 3. Stress-time Rhino-fodders in JWLS.

\begin{tabular}{|l|l|l|l|l|l|}
\hline $\begin{array}{c}\text { Sl. } \\
\text { No. }\end{array}$ & \multicolumn{1}{|c|}{ Plant Name } & \multicolumn{1}{|c|}{ Family } & \multicolumn{1}{|c|}{ Local Name } & \multicolumn{1}{|c|}{ Edible Parts } \\
\hline 1. & Bombax ceiba L. & Bombacaceae & Simul & Tree & Flower \\
\hline 2. & Mikania micrantha Kunth & Asteraceae & Assamlata, Mikenia & Climber & Leaves \\
\hline 3. & Typha angustifolia L. & Typhaceae & Hogla & $\begin{array}{l}\text { Rhizomatous } \\
\text { herb }\end{array}$ & Young shoot \\
\hline 4. & Vallisneria spiralis L. & Hydrocharitaceae & Jalua gachh & $\begin{array}{l}\text { Submerged } \\
\text { annual }\end{array}$ & Leaves \\
\hline
\end{tabular}

Table 4. Representation of different families among the different groups of Rhino-fodders in JWLS.

\begin{tabular}{|l|c|l|c|l|c|}
\hline Most Preferred Fodder & $\begin{array}{c}\text { No. of } \\
\text { Plants }\end{array}$ & Preferred Fodder & $\begin{array}{c}\text { No. of } \\
\text { Plants }\end{array}$ & $\begin{array}{c}\text { Stress-time } \\
\text { Fodder }\end{array}$ & $\begin{array}{c}\text { No. of } \\
\text { Plants }\end{array}$ \\
\hline Poaceae & 14 & Bignoniaceae & 1 & Asteraceae & 1 \\
\hline Zingiberaceae & 1 & Brassicaceae & 1 & Bombacaceae & 1 \\
\hline & & Cyperaceae & 5 & Hydrocharitaceae & 1 \\
\hline & & Euphorbiaceae & 3 & Typhaceae & 1 \\
\hline & & Fabaceae & 1 & & \\
\hline & & Hypoxidaceae & 1 & & \\
\hline & & Lauraceae & 1 & & \\
\hline & & Mimosaceae & 1 & & \\
\hline & & Moraceae & 1 & & \\
\hline & & Poaceae & 20 & & \\
\hline & & Sterculiaceae & 2 & & \\
\hline
\end{tabular}

Table 5. Families of Rhino-fodders in JWLS and their numerical representation at the species and variety level.

\begin{tabular}{|c|l|c|}
\hline Sl. No. & \multicolumn{1}{|c|}{ Families } & No. of Plants \\
\hline 1 & Poaceae & 34 \\
\hline 2 & Cyperaceae & 5 \\
\hline 3 & Euphorbiaceae & 3 \\
\hline 4 & Sterculiaceae & 2 \\
\hline 5 & Zingiberaceae & 2 \\
\hline 6 & Bombacaceae & 1 \\
\hline 7 & Asteraceae & 1 \\
\hline 8 & Bignoniaceae & 1 \\
\hline 9 & Brassicaceae & 1 \\
\hline 10 & Hydrocharitaceae & 1 \\
\hline 11 & Hypoxidaceae & 1 \\
\hline 12 & Lauraceae & 1 \\
\hline 13 & Mimosaceae & 1 \\
\hline 14 & Moraceae & 1 \\
\hline 15 & Papilionaceae & 1 \\
\hline 16 & Typhaceae & 1 \\
\hline & \multicolumn{2}{|c|}{ TOTAL: } \\
\hline
\end{tabular}


Chandra Ghosh and A.P. Das / Our Nature (2007)5: 14-20

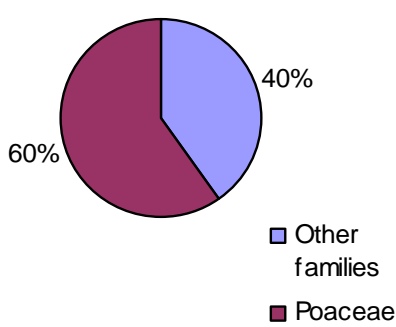

Figure 2. Grasses are dominating the fodder-flora for Rhinos.

grassland area in the sanctuary for the production of more fodder not only for Rhino but also for many other herbivore inhabitants. But whenever such plantation program is taken up certainly it is replacing other types of vegetation inside the sanctuary area. As it appears, sanctuary managers are not much concerned about the conservation of its rich biodiversity. Due to the use of mostly one particular species i.e., Saccharum narenga (Dhadda) the balance in the diversity of Rhino fodders is also much disturbed. Dhadda grows vigorously and, naturally, suppress the growth of other species in the vegetation. It is suggested that conservation policy or strategy for JWLS should also cover the idea of conserving all of species of plants and animals living there. They planted only single species of grass and due to its vigorous growth other species suppress.

\section{References}

Anonymous 1997. Management plan of Jaldapara Wildlife Sanctuary West Bengal. Wildlife Circle, Department of Forests, Govt. of West Bengal.

Banerjee, L.K. 1993. Plant resources of Jaldapara Rhino Sanctuary. Botanical Survey of India, Calcutta.

Champion, G.H. and S.K. Seth 1968. A revised survey of the forest types of India. Govt. of India, New Delhi.

Das, A.P., C. Ghosh and D. Bhowmick 2003. Project Report on estimation of Palatable Biomass in Jaldapara Wildlife Sanctuary with special reference to Rhinoceros unicornis $L$. Department of Botany, University of North Bengal, India.

Jain, S.K. and R.R. Rao 1977. A Handbook of Field and Herbarium Methods. Today \& Tomorrow's Printers and Publishers, New Delhi.

Pandit, P.K. 1996. Management plan of Jaldapara Wildlife Sanctuary (vols. I - III). Wildlife Circle, Department of Forests, Govt. of West Bengal.

Pandit, P.K., Chandra Ghosh and A.P. Das 2004. Non-timber Forest Produces from Jaldapara Wildlife Sanctuary: an Assessment. Indian Forester 130: $1169-1185$.

WII 1997. West Bengal World Bank Aides Forestry Project: Study on the Management of Rhinoceros (Final Report). Wildlife Institute of India, Dehradun. 